\title{
MiR93-5p inhibits chondrocyte apoptosis in osteoarthritis by targeting IncRNA CASC2
}

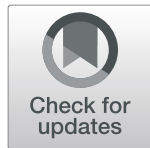

Yun Sun ${ }^{1}$, Simiao Kang ${ }^{2}$, Shuyan Pei ${ }^{1}$, Changmin Sang ${ }^{1}$ and Yijun Huang ${ }^{*^{*}}$ (D)

\begin{abstract}
Background: It has been reported that miR-93-5p and long non-coding RNA (IncRNA) Cancer Susceptibility 2 (CASC2) play opposite roles in regulating chondrocyte apoptosis, indicating the possible interaction between them. This study aimed to investigate the interaction between miR-93-5p and IncRNA CASC2 in chondrocyte apoptosis, which plays critical roles in osteoarthritis (OA).

Methods: The interaction between CASC2 and miR-93-5p was analyzed by dual luciferase assay and overexpression experiments. Levels of CASC2 and miR-93-5p in plasma sample from OA patients and healthy controls were measured by RT-qPCR. The roles of CASC2 and miR-93-5p in regulating the apoptosis of chondrocyte induced by LPS were analyzed by cell apoptosis assay.

Results: Through bioinformatics analysis we observed the potential interaction between CASC2 and miR-93-5p, which was confirmed by dual luciferase assay. In OA patients, miR-93-5p was downregulated, while CASC2 was upregulated, and they were inversely correlated. LPS treatment led to downregulated miR-93-5p and upregulated CASC2. Overexpression of miR-93-5p led to the downregulated CASC2 in chondrocytes. Under LPS treatment, CASC2 overexpression promoted the apoptosis of chondrocyte. MiR-93-5p overexpression played an opposite role and attenuated the effects of CASC2 overexpression.
\end{abstract}

Conclusion: MiR-93-5p was downregulated in OA may inhibit LPS-induced chondrocyte apoptosis by targeting InCRNA CASC2.

Keywords: Osteoarthritis, CASC2, miR-93-5p, Chondrocyte, Apoptosis

\section{Background}

Osteoarthritis (OA), also known as "wear and tear" arthritis or degenerative joint disease, mainly affect elderly with hips, knees and hands as the major affects sites [1]. $\mathrm{OA}$ is a major cause of chronic pain and disability, and a heavy burden on public health [2]. In some developed countries, the direct medical cost and indirect cost by reduced productivity causes the loss of 1 to $2.5 \%$ of the gross domestic product [3]. Aging, being female gender, obesity and occupational joint loading are the major risk factors for OA [4]. However, molecular pathogenesis of OA remains elusive $[5,6]$, leading to the difficulties in the development of novel therapeutic approaches.

\footnotetext{
*Correspondence: rj4607@163.com

${ }^{1}$ Department of Orthopaedics, Jiujiang University Clinical Medical

College-Jiujiang University Hospital, No.57 Xunyang East Road, Jiujiang City,

jiangxi Province 332000, People's Republic of China
}

Full list of author information is available at the end of the article
Previous studies have shown that genetic factors are critical players in the molecular pathogenesis of OA [7, 8]. Some signaling pathways, such as WNT signaling, have been proven to be potential targets for the development of targeted therapies [9]. Besides proteincoding genes, non-coding RNAs (ncRNAs), such as miRNAs and long (>200 nt) ncRNAs (lncRNAs), also regulate gene expression at multiple levels to participate in OA [10]. For instance, miR-93-5p inhibits the apoptosis of chondrocyte by targeting TCF4, thereby improving OA [11]. In contrast, IncRNA CASC2 promotes the apoptosis of chondrocytes to aggregate the conditions of OA [12]. The opposite functions of miR93-5p and CASC2 indicate the potential interactions between them in OA. In effect, our bioinformatics analysis also revealed the potential interaction between miR-93-5p and CASC2. This study was therefore

(c) The Author(s). 2020 Open Access This article is distributed under the terms of the Creative Commons Attribution 4.0 International License (http://creativecommons.org/licenses/by/4.0/), which permits unrestricted use, distribution, and reproduction in any medium, provided you give appropriate credit to the original author(s) and the source, provide a link to the Creative Commons license, and indicate if changes were made. The Creative Commons Public Domain Dedication waiver (http://creativecommons.org/publicdomain/zero/1.0/) applies to the data made available in this article, unless otherwise stated. 
carried out to analyze the potential interaction between miR-93-5p and CASC2 in OA.

\section{Methods \\ OA patients and controls}

Research subjects of this study included 60 OA patients (20 males and 40 females, 55 to 71 years, $63.0 \pm 5.3$ years) and 60 healthy controls (20 males and 40 females, 56 to 71 years, $63.3 \pm 5.1$ years) who were admitted to Jiujiang University Clinical Medical College, Jiujiang University Hospital between March 2016 and June 2019. This study passed the review of Ethics Committee of aforementioned hospital. OA patients' inclusion criteria: 1) patients who were diagnosed for the first time; 2) no therapies were initiated before admission. OA patients' exclusion criteria: 1) other clinical disorders were diagnosed; 2) recurrent OA. The $60 \mathrm{OA}$ patients included 27 cases of stage III and 33 cases of stage IV. According to the affected sites, there were 31 knee-affected cases and 29 hip-affected cases. The diagnosis of OA was performed through conventional methods, such as joint fluid analysis and X-ray imaging. Controls were enrolled to match the age and gender distributions of OA patients. All participants were informed of experimental design of this project. All patients and controls signed informed consent.

\section{Synovial fluid}

Before the initiation of therapies, extraction of synovial fluid from the affected sites was performed on all patients. To match OA patients, extraction of synovial fluid from knee was performed on 31 controls and extraction from hip was performed on the rest 29 cases. A liquid nitrogen sink was used to store the samples before use.

\section{Chondrocytes and transient transfections}

Primary chondrocytes (402OA-05A) from an OA adult were purchased from Sigma-Aldrich (USA) and were cultivated under conditions of $37^{\circ} \mathrm{C}$ with $5 \%$ $\mathrm{CO}_{2}$ in Chondrocyte Growth Medium (PromoCell). Cells were harvested at passage 5 to 7 to perform following experiments.

CASC2 expression vectors were constructed using pcDNA3.1 vector (Sangon) as backbone. Synthesis of miR-93-5p mimic and miRNA negative control (NC) was performed by Sangon. Lipofectamine 2000 Transfection Reagent (Invitrogen) was used to transfect $50 \mathrm{Nm}$ miRNA (miRNA NC as NC group) or $10 \mathrm{Nm}$ vector (empty vector as NC group) into 106 cells. Control@ cells were untransfected cells. The interval between following experiments and transfections was $24 \mathrm{~h}$.

\section{Luciferase reporter assay}

CASC2 full length cDNA was cloned into pGL3 plasmids (Promega). Through the aforementioned methods, cells were transfected with CASC2 vector+ miR-93-5p mimic or CASC2 vector+miRNA NC. Luciferase activity was measured by Dual Luciferase Reporter Assay Kit (Promega Corporation) using cells harvested at $24 \mathrm{~h}$ post-transfection.

\section{RNA samples and qPCR}

RNAiso Plus kit (Takara) was used to extract total RNA from synovial fluid specimens and in vitro cultivated cells. To harvest miRNAs, $85 \%$ was used to precipitate and wash RNA samples. To remove genomic DNA, all RNA samples were digested with DNA eraser at $37^{\circ} \mathrm{C}$ for $1 \mathrm{~h}$. In cases of LPS treatment, chondrocytes were treated with LPS at a concentration of 0, 200, 500 and $100 \mathrm{ng}$ for $24 \mathrm{~h}$ before use.

Total RNA reverse transcriptions (RTs) were performed using PrimeScript RT Master Mix (Takara) with total RNA as template to synthesize cDNA samples. With cDNA samples as template, qPCR mixtures were prepared using SYBR Premix Ex TaqTM II (Takara, Japan) to measure the expression levels of CASC2. $18 \mathrm{~S}$ rRNA was used as the endogenous control of CASC2.

To measure the expression levels of mature miR-93$5 \mathrm{p}$, polyadenylation, RTs and qPCR mixtures were performed using All-in-One ${ }^{\text {Tw }}$ miRNA qRT-PCR Reagent Kit (Genecopoeia). U6 was used as the endogenous control of miR-93-5p.

PCR reactions were performed in 3 replicates and fold-changes of gene expression were calculated using $2^{-\Delta \Delta \mathrm{Ct}}$ method.

\section{Cell apoptosis analysis}

Cells were harvested at $24 \mathrm{~h}$ post-transfection and cell suspensions $\left(3 \times 10^{5} / \mathrm{ml}\right)$ were prepared using nonserum cell culture medium. Cells were seeded onto a six-well plate with $2 \mathrm{ml}$ cell suspension per well, followed by the addition of $1 \mu \mathrm{g} / \mathrm{mL}$ LPS. Following cell culture under aforementioned methods for $24 \mathrm{~h}$, Annexin V-FITC Apoptosis Detection Kit (Thermo Fisher Scientific) was used to detect apoptotic cells. FACSCalibur flow cytometer was used to perform flow cytometer and data were analyzed by CellQuest software (BD Biosciences).

\section{Western-blot}

RIPA solution (Invitrogen) was used to was used to isolate proteins from chondrocytes. Proteins were denatured in boiling water for $10 \mathrm{~min}$ and were separated by 10\% SDS-PAGE gel electrophoresis. PVDF membranes were used to transfer proteins and blocking was performed in fat-free milk (5\% in PBS) at room temperature 
for $90 \mathrm{~min}$. Primary antibodies included rabbit antihuman cleaved caspase 3 (ab49822, 1:1000; Abcam) and GAPDH (ab9485, 1: 1000, Abcam) at $4{ }^{\circ} \mathrm{C}$ for $15 \mathrm{~h}$, followed by incubation with IgG-HRP goat anti-rabbit secondary antibody (MBS435036, 1:1000, MyBioSource) at room temperature for $2 \mathrm{~h}$. Signals were produced using Amersham ECL Western Blotting Detection Reagent (GE Healthcare) and were normalized using Image J v1.46 software.

\section{Statistical analysis}

Means \pm standard deviation (SD) was used to express the data of 3 independent biological replicates involved in each experiment. Unpaired t test was used to compare 2 groups. Exploration of differences among multiple groups was performed using ANOVA (one-way) and Tukey test. Correlations were analyzed by linear regression. $p<0.05$ indicated statistically significant differences.

\section{Results}

CASC2 can directly interact with miR-93-5p

The interaction between CASC2 and miR-93-5p was predicted using an online RNA interaction program named IntaRNA (http://rna.informatik.uni-freiburg.de/ IntaRNA/Input.jsp). It was observed that CASC2 and miR-93-5p can form strong base pairing (Fig. 1a). Dual luciferase reporter assay was performed to further analyze the interaction between CASC2 and miR-93-5p. Comparing to chondrocytes transfected with CASC2 vector and miRNA NC, cells transfected with CASC2 vector and miR-93-5p mimic showed significantly lower relative luciferase activity (Fig. $1 \mathrm{~b}, p<0.05$ ).

\section{MiR-93-5p and CASC2 were inversely correlated in synovial fluid}

Levels of miR-93-5p and CASC2 in synovial fluid from both OA $(n=60)$ and healthy controls $(n=60)$ were measured using qPCR and compared by unpaired $t$ test. It was observed that, comparing to controls, miR-93-5p was significantly downregulated (Fig. 2a, $p<0.05$ ), while CASC2 was significantly upregulated (Fig. 2b, $p<0.05$ ) in OA group. Linear regression analysis showed that expression levels of CASC2 were significantly and inversely correlated with expression levels of miR-93-5p across both OA (Fig. 2c) and control (Fig. 2d) synovial fluid samples.
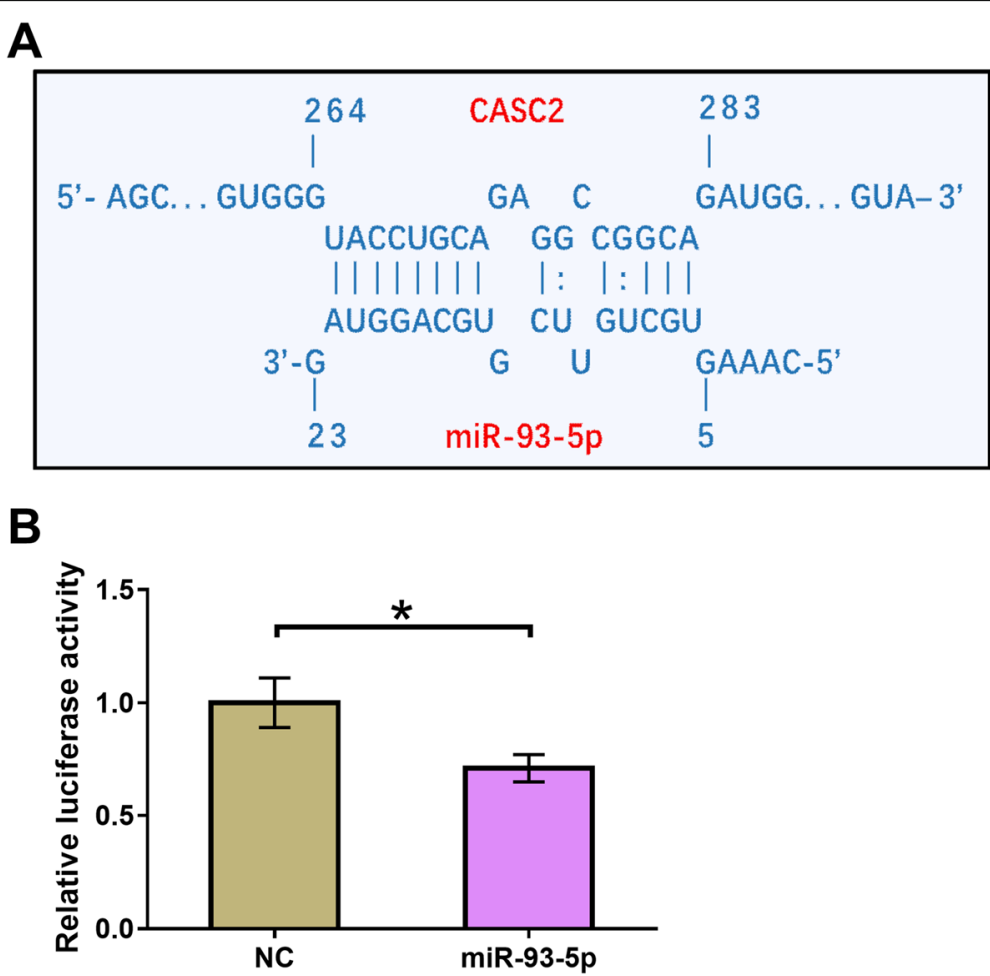

Fig. 1 CASC2 can directly interact with miR-93-5p. The interaction between CASC2 and miR-93-5p was predicted using an online RNA interaction program named IntaRNA (http://rna.informatik.uni-freiburg.de/IntaRNA/Input.jsp). It was observed that CASC2 and miR-93-5p can form strong base pairing (A). Dual luciferase reporter assay was performed by transfecting CASC2 vector and miRNA NC or CASC2 vector and miR-93-5p mimic into chondrocytes. Relative luciferase activity was compared by unpaired $t$ test. Experiments were repeated 3 times and mean values were presented.., $p<0.05$ 

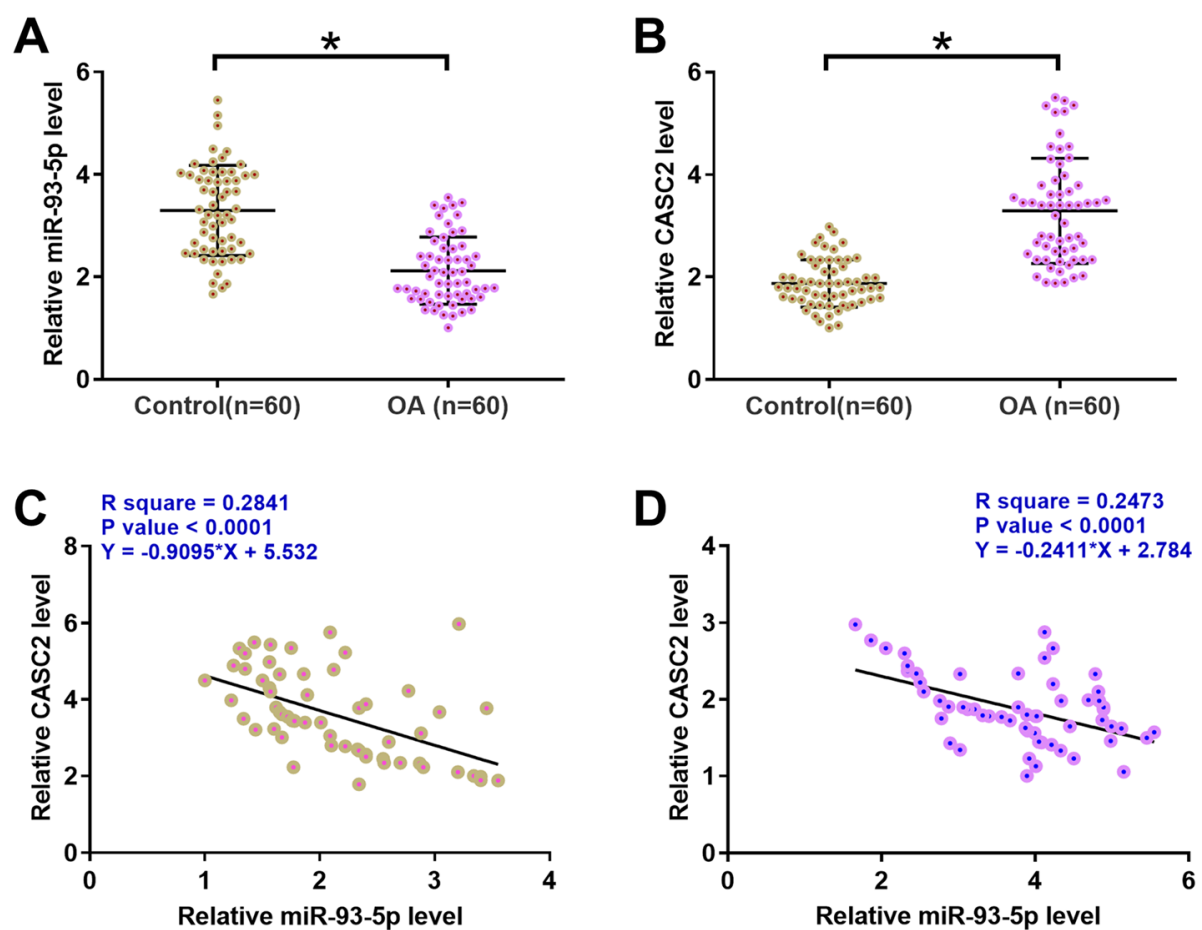

Fig. 2 MiR-93-5p and CASC2 were inversely correlated in synovial fluid. Levels of miR-93-5p (A) and CASC2 (B) in synovial fluid from both OA $(n=60)$ and healthy controls $(n=60)$ were measured using qPCR and compared by unpaired t test. PCR reactions were performed 3 times and mean values were presented. ${ }^{*}, p<0.05$. Linear regression was performed to analyze the correlation between expression levels of CASC2 and miR-93-5p across both OA (C) and control (D) synovial fluid samples
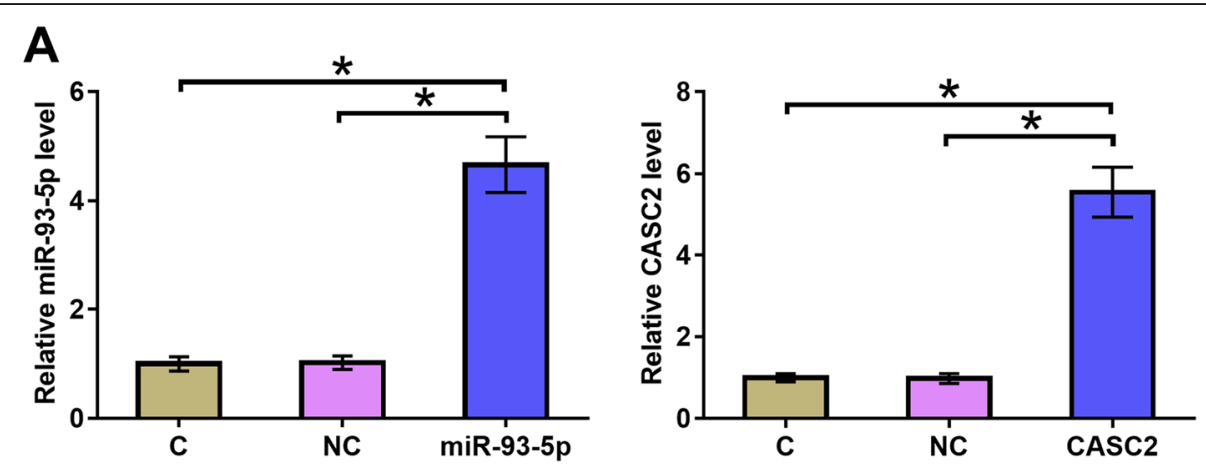

B

C
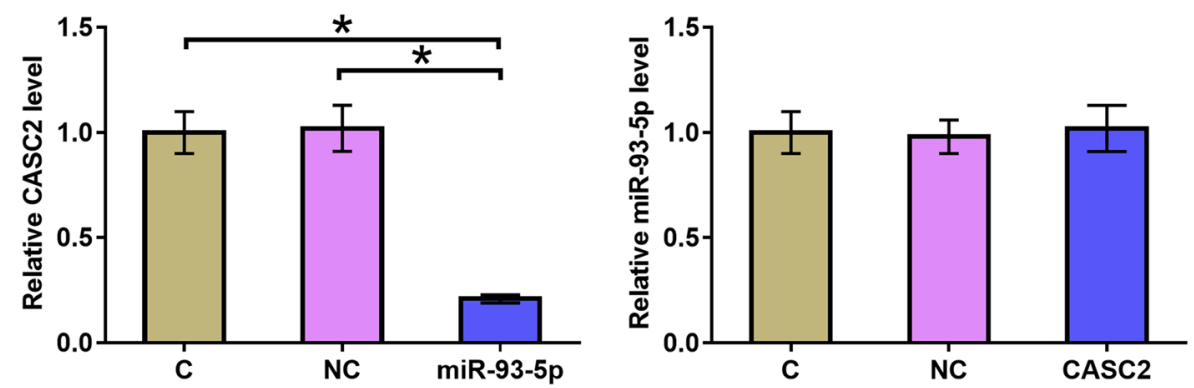

Fig. 3 Overexpression of miR-93-5p led to the downregulated CASC2 in chondrocytes. Chondrocytes were transfected with miR-93-5p mimic and CASC2 expression vector. Overexpression of miR-93-5p and CASC2 was confirmed by qPCR at $24 \mathrm{~h}$ post-transfection (a). The effects of miR-93-5p overexpression on CASC2 (b) and the effects of CASC2 overexpression on miR-93-5p (c) were also analyzed by qPCR. Experiments were repeated 3 times and mean values were presented.*, $p<0.05$ 


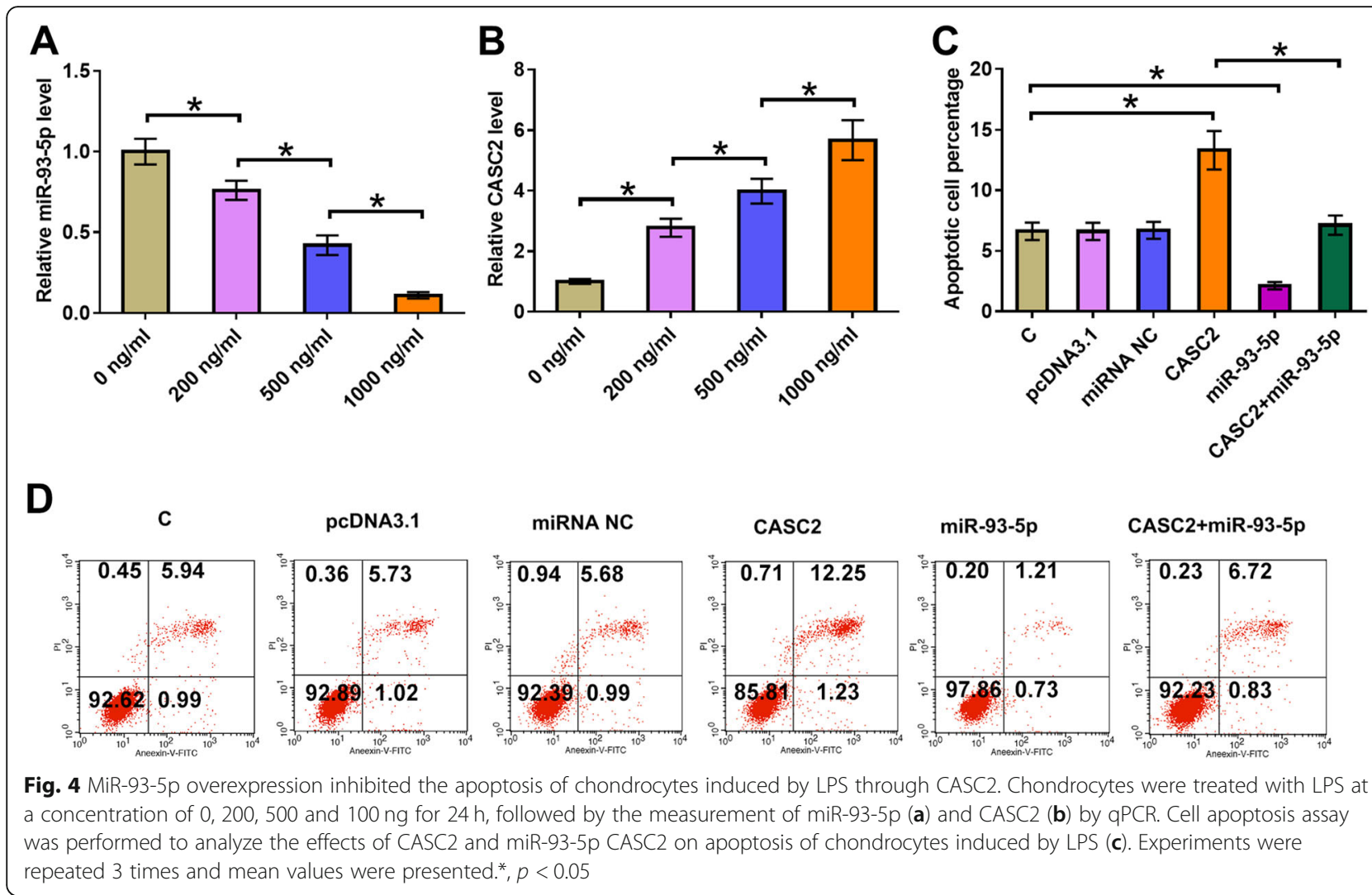

\section{Overexpression of miR-93-5p led to the downregulated CASC2 in chondrocytes}

Chondrocytes were transfected with miR-93-5p mimic and CASC2 expression vector. Overexpression of miR93-5p and CASC2 was confirmed by qPCR at $24 \mathrm{~h}$ posttransfection (Fig. 3a, $p<0.05$ ). Comparing to $\mathrm{C}$ and $\mathrm{NC}$ groups, overexpression of miR-93-5p led to downregulated CASC2 (Fig. 3b, $p<0.05$ ). In contrast, CASC2 overexpression failed to significantly affect the expression of miR-93-5p (Fig. 3c, $p<0.05$ ).

\section{MiR-93-5p overexpression inhibited the apoptosis of chondrocytes induced by LPS through CASC2}

Chondrocytes were treated with LPS at a concentration of $0,200,500$ and $100 \mathrm{ng}$ for $24 \mathrm{~h}$, followed by the measurement of miR-93-5p and CASC2 by qPCR. It was observed that LPS treatment led to downregulated miR-93-5p (Fig. 4a, $p<0.05$ ) and upregulated CASC2 (Fig. 4b, $p<0.05$ ) in a dose-dependent manner. Cell apoptosis assay was performed to analyze the effects of CASC2 and miR-93-5p CASC2 on apoptosis of chondrocytes induced by LPS. Comparing to $\mathrm{C}$ group, CASC2 overexpression promoted the apoptosis of chondrocyte. MiR-93-5p overexpression played an opposite role and attenuated the effects of CASC2 overexpression (Fig. 4c, $p<0.05$ ). Consistently, CASC2 overexpression led to increased level of cleaved caspase 3. MiR-93-5p overexpression played an opposite role and attenuated the effects of CASC2 overexpression on the production of cleaved caspase 3 (Fig. $5, p<0.05$ ).

\section{Cleaved caspase 3}

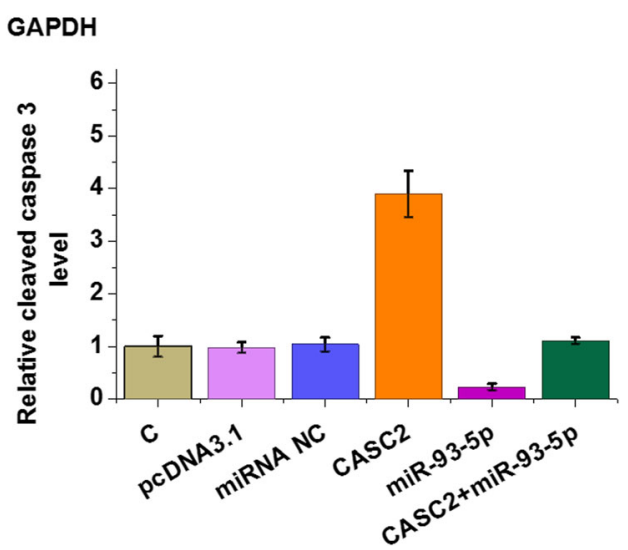

Fig. 5 MiR-93-5p overexpression suppressed the production of cleaved caspase 3 in chondrocytes through CASC2. Western blot was performed to analyze the effects of CASC2 and miR-93-5p CASC2 on the production of cleaved caspase 3 in chondrocytes treated by LPS (C). Experiments were repeated 3 times and representative images were presented.*, $p<0.05$ 


\section{Discussion}

This study mainly explored the interaction between miR93-5p and CASC2 and their roles in OA. We first characterized CASC2 as a downstream target of miR-93-5p.

CASC2 is a well-characterized tumor suppressive lncRNAs in many different types of cancers [13, 14]. CASC2 participate in cancer biology mainly by regulating cancer cell behaviors, such as inhibiting cell proliferation and promoting cell apoptosis $[13,14]$. The development of OA leads to cartilage damage, while the only cells in healthy cartilage are chondrocytes [15]. In effect, the apoptosis of chondrocytes plays a major role in the pathogenesis of OA [15]. A recent study reported that CASC2 was upregulated in $\mathrm{OA}$ and promoted chondrocyte apoptosis to aggregate disease conditions [12]. Consistently, our study also observed increased apoptotic rate of chondrocytes under LPS treatment after CASC2 overexpression. Our data and previous studies showed that CASC2 may play similar roles in regulating the apoptosis of different types of cells to participate in different types of diseases.

Inhibition of chondrocyte apoptosis is considered as a promising target for the treatment of OA [16]. In a recent study, $\mathrm{Xu}$ et al. reported that miR-93-5p can target TCF4 to suppress cartilage degradation and the apoptosis of chondrocyte to improve the conditions of OA [11]. Our study used LPS-treated chondrocytes as the cell model of OA because LPS participates in diverse pathological processes involved in OA, ranging from inflammatory responses and cell apoptosis [17]. Consistent with previous studies, our study also showed the inhibitory effects of miR-93-5p on the apoptosis of chondrocytes induced by LPS.

The key finding of the present study is that miR-93-5p can directly target CASC2 to participate in the regulation of chondrocyte apoptosis. Therefore, miR-93-5p may have multiple downstream targets (TCF4 as aforementioned) in chondrocytes. Future studies are needed to explore other potential targets.

\section{Conclusion}

In conclusion, miR-93-5p is downregulated in OA and can directly target CASC2 to inhibit LPS-induced apoptosis of chondrocytes.

\section{Abbreviations transcription \\ Acknowledgements \\ Not applicable.}

C: Control; CASC2: Cancer Susceptibility 2; GAPDH: Glyceraldehyde 3phosphate dehydrogenase; IncRNA: long non-coding RNA;

LPS: Lipopolysaccharides; NC: Negative control; OA: Osteoarthritis; RT: Reverse

\section{Authors' contribution}

YS and YJH designed and carried out the study. SMK, SYP, CMS participated in experiments and statistical analysis. YS wrote the manuscript. YJH revised the manuscript. All authors have rad and approved the final manuscript.
Funding

Not applicable.

\section{Availability of data and materials}

The analyzed data sets generated during the study are available from the corresponding author on reasonable request.

\section{Ethics approval and consent to participate}

The present study was approved by the Ethics Committee of Jiujiang University Clinical Medical College, Jiujiang University Hospital. The research has been carried out in accordance with the World Medical Association Declaration of Helsinki. All patients and healthy volunteers provided written informed consent prior to their inclusion within the study.

\section{Consent for publication}

Not applicable.

\section{Competing interests}

The authors declare that they have no competing interests.

\section{Author details}

'Department of Orthopaedics, Jiujiang University Clinical Medical College.Jiujiang University Hospital, No.57 Xunyang East Road, Jiujiang City, jiangxi Province 332000, People's Republic of China. ${ }^{2}$ Department of

Orthopaedics, Harbin Children's Hospital, Harbin City, Heilong Jiang Province 150000, People's Republic of China.

Received: 23 September 2019 Accepted: 24 December 2019 Published online: 13 January 2020

\section{References}

1. Glyn-Jones S, AJR P, Agricola R, et al. Osteoarthritis. Lancet. 2015;386(9991): 376-87.

2. Allen KD, Golightly YM. Epidemiology of osteoarthritis: state of the evidence. Curr Opin Rheumatol. 2015;27(3):276-83.

3. Hiligsmann M, Cooper C, Arden N, et al. Health economics in the field of osteoarthritis: an expert's consensus paper from the European Society for Clinical and Economic Aspects of osteoporosis and osteoarthritis (ESCEO) [C]//seminars in arthritis and rheumatism. WB Saunders. 2013;43(3):303-13.

4. Palazzo C, Nguyen C, Lefevre-Colau MM, et al. Risk factors and burden of osteoarthritis. Ann Phys Rehabil Med. 2016;59(3):134-8.

5. Hedbom E, Häuselmann HJ. Molecular aspects of pathogenesis in osteoarthritis: the role of inflammation. Cell Mol Life Sci. 2002;59(1):45-53.

6. Krasnokutsky S, Attur M, Palmer G, et al. Current concepts in the pathogenesis of osteoarthritis. Osteoarthr Cartil. 2008;16:S1-3.

7. Sandell LJ. Etiology of osteoarthritis: genetics and synovial joint development. Nat Rev Rheumatol. 2012;8(2):77-89.

8. Warner $\mathrm{S}$, Valdes $\mathrm{A}$. The genetics of osteoarthritis: A review. J Funct Morphol Kinesiol. 2016;1(1):140-53.

9. Blom $A B$, van Lent $L P$, van der Kraan MP, et al. To seek shelter from the WNT in osteoarthritis? WNT-signaling as a target for osteoarthritis therapy. Curr Drug Targets. 2010;11(5):620-9.

10. Barter MJ, Young DA. Epigenetic mechanisms and non-coding RNAs in osteoarthritis. Curr Rheumatol Rep. 2013;15(9):353.

11. Xue H, Tu Y, Ma T, et al. miR-93-5p attenuates IL-1 $\beta$-induced chondrocyte apoptosis and cartilage degradation in osteoarthritis partially by targeting TCF4. Bone. 2019:123:129-36.

12. Huang T, Wang J, Zhou Y, et al. LncRNA CASC2 is up-regulated in osteoarthritis and participates in the regulation of $\mathrm{IL}-17$ expression and chondrocyte proliferation and apoptosis. Biosci Rep. 2019;39(5): BSR20182454.

13. Fan JC, Zeng F, Le YG, et al. LnCRNA CASC2 inhibited the viability and induced the apoptosis of hepatocellular carcinoma cells through regulating miR-24-3p. J Cell Biochem. 2018;119(8):6391-7.

14. Li P, Xue WJ, Feng $Y$, et al. Long non-coding RNA CASC2 suppresses the proliferation of gastric cancer cells by regulating the MAPK signaling pathway. Am J Transl Res. 2016;8(8):3522.

15. Hwang $\mathrm{H}$, Kim H. Chondrocyte apoptosis in the pathogenesis of osteoarthritis. Int J Mol Sci. 2015;16(11):26035-54. 
16. Hosseinzadeh A, Kamrava SK, Joghataei MT, et al. Apoptosis signaling pathways in osteoarthritis and possible protective role of melatonin. J Pineal Res. 2016;61(4):411-25.

17. Huang Z, Stabler T, Pei F, et al. Both systemic and local lipopolysaccharide (LPS) burden is associated with knee osteoarthritis (OA). Osteoarthr Cartil. 2016;24:S329-30.

\section{Publisher's Note}

Springer Nature remains neutral with regard to jurisdictional claims in published maps and institutional affiliations.

Ready to submit your research? Choose BMC and benefit from:

- fast, convenient online submission

- thorough peer review by experienced researchers in your field

- rapid publication on acceptance

- support for research data, including large and complex data types

- gold Open Access which fosters wider collaboration and increased citations

- maximum visibility for your research: over $100 \mathrm{M}$ website views per year

At BMC, research is always in progress.

Learn more biomedcentral.com/submissions 